\section{Comment on Tocheva et al. "Sporulation, bacterial cell envelopes and the origin of life"}

\author{
Iain C. Sutcliffe and Lynn G. Dover
}

In their recent Opinion article (Sporulation, bacterial cell envelopes and the origin of life. Nat. Rev. Microbiol. 14, 535-542 (2016)) ${ }^{1}$, Tocheva and colleagues build on their elegant electron cryotomography studies to derive an intriguing hypothesis that sporulation in a primordial monoderm bacterium (having a single membrane) could have resulted in the evolution of sporulating diderm bacteria (having a double membrane) and thus all extant bacteria. The evolution of outer membranes as a consequence of the topography of sporulation is a plausible hypothesis; however, it should be highlighted that Tocheva et al. consider a relatively limited range of the known and candidate phyla in their model. Thus, it will be of interest to see how their hypothesis stands up when tested against expanded phylogenetic analyses; for example, including the many new phyla that were identified in recent large-scale genomic analyses, which include novel monoderm phyla and possibly phyla with novel cell envelope architectures ${ }^{2,3}$.

Although the hypothesis of Tocheva et al. ${ }^{1}$ may help to explain the evolution of most types of outer membrane, we question their suggestions that all diderm bacteria have arisen from an ancestral sporulating diderm and that outer membranes have probably only evolved once. As briefly acknowledged by Tocheva et al., the uniqueness of the mycolic acid-based outer membranes (MOMs) of members of the suborder Corynebacterineae challenges these assumptions. The 'mycolata' are significant because they belong to the predominantly monoderm phylum Actinobacteria and contain several major pathogens, including Mycobacterium tuberculosis. Several lines of evidence support the uniqueness of the MOM. First, mycolic acids are structurally distinct, being formed from condensed fatty acids ${ }^{4}$. Second, in contrast to the outer membranes of all other diderm bacteria, mycolic acids are the only outer-membrane lipids that are described as being covalently anchored to the underlying arabinogalactan-peptidoglycan ${ }^{4,5}$, which results in a unique architecture that is unlikely to have arisen from remodelling of typical outer membranes, as suggested by Tocheva et al. ${ }^{1}$ Third, all other characterized outer membranes contain BamA family proteins, which have a central role in $\beta$-barrel protein insertion, along with several specialized protein secretion systems ${ }^{5,6}$. It is notable that neither BamA proteins nor type I-VI secretion systems have been identified in any mycolata $^{5-7}$. Although bioinformatic evidence suggests that proteins with high $\beta$-sheet content are found in the MOM, relatively few MOM proteins have been characterized in detail, and orthology with $\beta$-barrels in other membranes cannot be assumed. Notably, the structures of MOM porins differ from the canonical $\beta$-barrels of porins in other diderm $\operatorname{taxa}^{8}$. The homology of the carboxyl terminus of mycobacterial Rv0899 with proteobacterial outer-membrane protein A (OmpA) proteins (highlighted by Tocheva et al. ${ }^{1}$ ), seems to reflect its function as a periplasmic peptidoglycanbinding domain, but its overall topology and location remain unclear ${ }^{9}$.

Cumulatively, these observations argue against a common evolutionary history for the MOM of Corynebacterineae and the outer membranes in other diderm bacteria. Thus, we argue, in contrast to the conclusions of Tocheva et al. ${ }^{1}$, that structurally distinct outer membranes probably evolved at least twice.

Iain C. Sutcliffe and Lynn G. Dover are at the Faculty of Health and Life Sciences, Northumbria University, Newcastle Upon Tyne NE1 8ST, UK.

Correspondence to I.C.S. iain.sutcliffe@northumbria.ac.uk

doi:10.1038/nrmicro2016.113 Published online 25 Jul 2016

1. Tocheva, E. I., Ortega, D. R. \& Jensen, G. J. Sporulation, bacterial cell envelopes and the origin of life. Nat. Rev. Microbiol. 14, 535-542 (2016).

2. Albertsen, M. et al. Genome sequences of rare, uncultured bacteria obtained by differential coverage binning of multiple metagenomes. Nat. Biotechnol. 31, 533-538 (2013).

3. Hug, L. A. et al. A new view of the tree of life Nat. Microbiol. 1, 16048 (2016).

4. Dover, L. G., Cerdeno-Tarraga, A. M., Pallen, M. J., Parkhill, J. \& Besra, G. S. Comparative cell wall core biosynthesis in the mycolated pathogens, Mycobacterium tuberculosis and Corynebacterium diphtheriae. FEMS Microbiol. Rev. 28, 225-250 (2004).

5. Sutcliffe, I. C. A phylum level perspective on bacterial cell envelope architecture. Trends Microbiol. 18, 464-470 (2010).

6. Sutcliffe, I. C. Cell envelope architecture in the Chloroflexi: a shifting frontline in a phylogenetic turf war. Environ. Microbiol. 13, 279-282 (2011).

7. Abby, S. S. et al. Identification of protein secretion systems in bacterial genomes. Sci. Rep. 6, 23080 (2016).

8. Niederweis, M., Danilchanka, O., Huff, J., Hoffmann, C. \& Engelhardt, H. Mycobacterial outer membranes: in search of proteins. Trends Microbiol. 18, 109-116 (2010).

9. Yao, Y., Barghava, N., Kim, J., Niederweis, M ¿ Marassi, F. M. Molecular structure and peptidoglycan recognition of Mycobacterium tuberculosis ArfA (Rv0899). J. Mol. Biol. 416, 208-220 (2012)

Competing interests statement

The authors declare no competing interests. 